\title{
EDITORIAL
}

\section{PERCEPCIÓN DE LA CONTINUIDAD ASISTENCIAL. CONOCER PARA ACTUAR}

\author{
Antonio Fernández Moyano (1) y Manuel Ollero Baturone (2) \\ (1) Servicio de Medicina Interna. Hospital S. Juan de Dios del Aljarafe. Sevilla \\ (2) Unidad Clínica de Atención Medica Integral. Hospital Universitario Virgen del Rocio. Sevilla.
}

La coordinación asistencial es la capacidad de los servicios sanitarios para ofertar sincronizadamente un objetivo asistencial común sin que se produzcan desencuentros que perjudiquen al paciente ${ }^{1}$. La continuidad asistencial según la definición clásica ${ }^{2}$ es la percepción que tiene el usuario sobre la coherencia de esta asistencia coordinada, que recibe a lo largo del tiempo. Freeman ${ }^{3}$, posteriormente, introdujo varios conceptos operativos que deben integrarse en esta definición, como son el ámbito de la existencia identificada de responsable asistencial, de la longitudinalidad del seguimiento en el tiempo, de la flexibilidad para adaptarse a los cambios personales y sociales y finalmente de la información efectiva con el paciente, cuidadores y otros profesionales.

La coordinación asistencial está identificada como herramienta de gestión clínica generadora de mejor calidad de vida y satisfacción de los usuarios. Esto ha hecho que los sistemas sanitarios la integren en los distintos planes de calidad para enfermedades crónicas complejas, de forma que figuren transversalmente en sus formas de trabajo y se incluya en la formación de los profesionales ${ }^{4,5}$.

Hasta hace escasamente un año no se había abordado la identificación sistemática de los distintos factores relacionados con la continuidad asistencial. En 2009 el National
Institutes of Health ${ }^{6}$ publicó los resultados del programa de investigación sobre la continuidad de la atención sanitaria, donde se identificaron los factores percibidos por usuarios, cuidadores y profesionales que se asociaban a ésta. No obstante la valoración realizada era fundamentalmente cualitativa, de forma que la cuantitativa solo había sido comunicada en pacientes con cáncer, diabetes mellitus, enfermedad vascular cerebral y enfermedades psiquiátricas severas.

Tampoco se había evaluado - una vez identificados cuantitativa o cualitativamente estos factores- la influencia que su modificación pudiera tener en la percepción de la continuidad asistencial en distintos sistemas sanitarios. Ha sido muy recientemente cuando se ha publicado la experiencia tras la modificación de algunas variables asociadas a continuidad asistencial en dos regiones de la provincia de Quebec $^{7}$. En este estudio, con un diseño de comparación antes-después tras modificar algunos de estos parámetros, se presenta la valoración cuantitativa de los usuarios sobre la continuidad asistencial, encontrando una mejora de la percepción en algunos de los indicadores. Esta evaluación fue realizada a través de indicadores obtenidos de un cuestionario de validación de los cuidados, que estaban centrados casi exclusivamente en atención primaria (AP) y no en atención hospitalaria (AH), lo que limita su aplicabilidad ${ }^{8}$. 
Así, hasta ahora no disponíamos de una evaluación cuantitativa del efecto de los distintos factores asociados a la continuidad asistencial realizada a pacientes -entre los que se incluyen los que tienen enfermedades crónicas complejas-, y que hayan sido atendidos en distintos ámbitos sanitarios durante un razonable periodo de tiempo. Tampoco se había evaluado con estas características la percepción de las distintas dimensiones de continuidad asistencial (relación, información y gestión).

En este número de Revista Española de Salud Pública Aller y colaboradores ${ }^{9}$ abordan en el escenario asistencial real y diario la evaluación cuantitativa por parte de los usuarios de los factores que se relacionan con la continuidad asistencial, y los distintos grados alcanzados en las dimensiones referidas de ésta, validando paralelamente el cuestionario utilizado ${ }^{10}$.

Los resultados obtenidos son destacables. Como mejor percibidos está que la mayoría de los usuarios - con una puntuación media aproximada de 8,5 sobre 10 tiene confianza en los médicos que le atienden, considerando adecuada su comunicación con los pacientes y recomendándolos a sus allegados. Se identifica una elevada consistencia profesional ya que la mayoría de usuarios fueron atendidos por el mismo profesional de AP y de AH. Finalmente gran parte consideró que los niveles asistenciales estaban razonablemente coordinados, fundamentado sobre todo en la comunicación entre profesionales, aunque la atribución en un $35 \%$ a la conformidad con la atención recibida pudo sobrevalorar este resultado.

Como aspectos con percepción más negativa resaltan la limitada transferencia de información entre niveles, pero sobre todo de AP a AH. Hasta el 20-30 \% de las personas encuestadas percibía deficiencias en la transferencia de la información clínica desde $\mathrm{AP}$ a $\mathrm{AH}$, mientras que esta percepción defi- citaria mejoraba hasta no sobrepasar el $15 \%$ cuando la transferencia de información era a la inversa. Hay poca contratransferencia desde AH a AP tras la evaluación, de forma que casi la mitad de los encuestados indicaban que el medico de AH no los volvió a derivar al medico de familia tras la visita. Además, sólo una minoría (26,5 \%) considera que tanto el medico de familia como el de AH conjuntamente eran los responsables de la gestión de su motivo de consulta, siendo mayoritaria (54\%) la consideración en la que el único responsable es el médico del hospital. Finalmente casi el $40 \%$ de los usuarios considera excesiva la demora de la primera consulta en $\mathrm{AH}$.

La relevancia del trabajo de Aller y colaboradores ${ }^{9}$ reside fundamentalmente en haber realizado una valoración cuantitativa de la continuidad asistencial en un ámbito asistencial real de nuestro entorno, identificando las distintas dimensiones que la conforman. Es muy relevante la amplitud etaria y de patologías de la población evaluada, lo que le otorga razonable validez externa. Hubiera sido deseable no obstante, poder diferenciar más rangos etarios en la parte superior de estos, estratificar funcionalmente la enfermedad crónica de base e identificar la existencia de pluripatología, ya que estas características basales condicionan -más en sí que el numero- parte de las necesidades asistenciales, lo que conlleva la intensidad de la utilización de recursos y por tanto las posibilidades de su valoración. En relación a estas necesidades asistenciales hay que reflexionar sobre, si para las personas con enfermedades crónicas complejas, los resultados funcionales pueden ser menos importantes a medida que pasa el tiempo y las cuestiones de un sentido de sí mismo, y de consistencia en la responsabilidad del proceso asistencial pueden llegar a ser más importantes. Tal como indican los autores, un próximo estudio más amplio nos podrá aproximar a estas cuestiones. 
Este original, es importante porque por una parte permite proponer oportunidades de mejora, como la posibilidad de incorporar valoraciones no presenciales que permitiesen mejorar la percepción de demora excesiva en la atención hospitalaria, el avance hacia la historia clínica digital compartida, o la necesidad de incorporar los cuidados de enfermería a las herramientas de evaluación de la continuidad asistencial entre otros. Por otra, porque el cuestionario presentado va a servir como sistema de evaluación cuantitativo de la continuidad asistencial en los distintos modelos organizativos.

En definitiva, las estrategias de mejora de la continuidad asistencial sigue siendo un reto no conseguido que tienen los Sistemas Sanitarios Públicos. Por ello es necesario avanzar decididamente en las políticas de coordinación entre los distintos niveles sanitarios y sociales -formales e informales-, que integran los procesos asistenciales; de tal manera que el ciudadano perciba continuidad en el proceso diagnóstico, de tratamiento y de cuidados. Los resultados presentados y la validación realizada del cuestionario, son una excelente primera parte necesaria, que nos abrirá la posibilidad de una evaluación más completa de la continuidad asistencial en distintas situaciones clínicas y ámbitos organizativos. A partir de ahora será más fácil conocer para poder actuar.

\section{BIBLIOGRAFIA}

1. Starfield B. Coordinación de la atención en salud. En: Starfield B, editor. Atención primaria. Barcelona: Masson; 2002. p. 233-65.

2. Haggerty J, Reid R, Freeman GK, Starfield BH, Adair CE, McKendry R. Continuity of care: a multidisciplinary review. BMJ. 2003; 327:1219-21.
3. Freeman GK, Olesen F and Hjortdahl P. Continuity of care: an essential element of modern general practice? Family Practice 2003; 20: 623-627.

4. II Plan de Calidad del sistema sanitario Público de Andalucia 2005-2008. Consejeria de Salud. Disponible en http://www.juntadeandalucia.es/salud/ sites/csalud/galerias/ documentos /c 1 c 6 planes_estrategias/II_plan_calidad/II_plan_calidad.p df (Accedido en Septiembre del 2010).

5. Marco estratégico para la mejora de la atencion primaria en España. Proyecto AP-21. Ministerio de Sanidad y Politica Social 2007-2010. Disponible en http://www.msps.es/profesionales/proyectosActividades/docs/AP2 1 MarcoEstrategico2007_2012.pdf ( Accedido en Septiembre del 2010).

6. Parker G, Corden A, Heaton J. Synthesis and conceptual analysis of the SDO Programme's research on continuity of care. Report for the National Institute for Health Research Service Delivery Organisation programme. National Institute for Health Research Evaluations, Trials and Studies Coordinating Centre, Southampton; 2009. Disponible en http://php.york.ac.uk/inst/spru/pubs/1241/. (Accedido en Septiembre del 2010).

7. Tourigny A, Aubin M, Haggerty J, Bonin L, Morin D, Reinharz D, Leduc Y, St-Pierre M, Houle N, Giguère A, Benounissa Z, Carmichael PH.. Patients' perceptions of the quality of care after primary care reform. Can Fam Physician. 2010 Jul;56(7):e273-82.

8. Shi L, Starfield B, Xu J. Validating the adult Primary Care Assessment Tool. J Fam Pract 2001; 50(2):161.

9. Aller Hernández MB, Vargas Lorenzo I, SánchezPérez I, Henao Martínez D, Coderch de Lassaletta J, Llopart López JR, Ferran Mercadé M, Colomés Figuera Ll y Vázquez Navarrete ML. La continuidad asistencial entre niveles percibida por usuarios del sistema de salud en Cataluña. Rev Esp Salud Publica. 2010; 84: 371-387.

10. Letelier MJ, et al. Diseño y validación de un cuestionario para medir la continuidad asistencial entre niveles desde la perspectiva del usuario: CCAENA. Gac Sanit. 2010. doi: 10.1016/ j.gaceta.2010. 03.010 